\title{
REFLEXIONES SOBRE EL PAPEL Y DISEÑO DE LOS DICCIONARIOS DE TRADUCCIÓN ESPECIALIZADA
}

\author{
Sven Tarp \\ Universidad de Aarhus (Dinamarca) \\ st@asb.dk
}

\section{Resumen}

Este artículo trata de los diccionarios especializados de traducción. Basado en los principios de la teoría funcional, analiza las diversas fases y subfases del proceso traductivo desde una perspectiva lexicográfica mostrando que un diccionario de traducción, si realmente pretende resolver las complejas necesidades de sus usuarios, debe ser mucho más que un simple diccionario bilingüe. A continuación presenta un concepto global de diccionario de traducción que incluye diversos componentes monolingües y bilingües en ambas direcciones entre las dos lenguas en cuestión. Finalmente, el artículo debate cómo este concepto puede aplicarse en Internet con el fin de desarrollar diccionarios de traducción de alta calidad y rápido acceso a datos adaptados cada vez más a las necesidades de cada traductor.

\begin{abstract}
"Reflection on the role and design of specialised translation dictionaries"

This article deals with specialised translation dictionaries. Based on the principles of the function theory, it analyses the different phases and sub-phases of the translation process from a lexicographical perspective and shows that a translation dictionary should be much more than a mere bilingual dictionary if it really aims to meet its users' complex needs. Thereafter, it presents a global concept of a translation dictionary which includes various mono- and bilingual components in both language directions. Finally, the article discusses how this concept can be applied on the Internet in order to develop high-quality translation dictionaries with quick access to data that are still more adapted to the needs of each translator.
\end{abstract}


Palabras clave: Lexicografía especializada. Diccionarios de traducción. Diccionarios en línea. Teoría funcional. Proceso de traducción.

Keywords: Specialised lexicography. Translation dictionaries. Online dictionaries. Function theory. Translation process. 


\section{Introducción}

Hay mitos -muchos mitos- sobre la relación entre lexicografía y traducción. Uno de los más enraizados y repetidos es el que concibe los diccionarios bilingües y de traducción como casi sinónimos o, por lo menos, considera que este último tipo de diccionario siempre es bilingüe; cf. Bergenholtz et al. (1997), Burkhanov (1998), Hartmann \& James (2001) y Marello (2003). Con esta contribución quiero mostrar que la realidad es mucho más compleja, en línea con lo que afirmaba Piotrowski (1994), uno de los pocos lexicógrafos que ha estudiado esta problemática de una forma más completa.

Este artículo se basa en la teoría de las funciones lexicográficas. Según esta teoría, los diccionarios y demás obras lexicográficas son herramientas de información, por lo que deben ser las necesidades de información que puedan tener usuarios potenciales en diversos contextos y situaciones, las que, en última instancia, determinen las características de un proyecto de diccionario. La teoría funcional, además, considera que la lexicografía es una disciplina independiente con su propio sistema de teorías, métodos, etc., disciplina que, al mismo tiempo, tiene una gran vocación interdisciplinaria que, a lo largo de la historia, la ha relacionado con casi todas las actividades y áreas de pensamiento del ser humano; cf. Tarp (2008) y Fuertes Olivera \& Tarp (2014).

Por su parte, la teoría -o las teorías- de la traducción se ha desarrollado enormemente durante las últimas décadas y ha logrado mostrar la gran complejidad que caracteriza el proceso de traducción con todas sus fases y subfases de tipo cognitivo-mental y práctico; cf. Snell-Hornby et al. (1992), Bassnett (2001), Gerzymisch-Arbogast et al. (2008), entre muchos otros. La lexicografía puede inspirarse en estos avances a la hora de comprender el proceso de traducción desde una perspectiva teórica; sin embargo, no debe copiar tales avances sin una reflexión crítica previa, algo que es fundamental, como veremos en este artículo.

Como disciplina independiente, la lexicografía debe estudiar los resultados de otras disciplinas relevantes para determinar lo que debe rechazarse, lo que puede incorporarse sin más y lo que solo puede incorporarse una vez adaptado a los requerimientos y características de la lexicografía. Este es un 
principio fundamental de metodología que, lamentablemente, no siempre se aplica, con las subsiguientes consecuencias, muy negativas para los diccionarios y, por ende, para los usuarios.

Por lo tanto, lo que interesa a la lexicografía son únicamente las fases y subfases del proceso de traducción donde pueden producirse problemas y necesidades lexicográficamente relevantes, es decir, problemas y necesidades que pueden resolverse mediante una consulta a un diccionario. A continuación vamos a estudiar el proceso de traducción desde el punto de vista de la lexicografía especializada que, por sus características, ofrece una visión aún más amplia que la lexicografía general de cómo se expresa la compleja relación que existe entre lexicografía y traducción. Empezamos por una pequeña excursión por el mundo de la metodología.

\section{Métodos aplicados y aplicables en la lexicografía especializada}

En la mayoría de los proyectos en los que supuestamente se prepara un diccionario especializado, se copian sencillamente conceptos de diccionarios ya existentes sin profundizar en las necesidades reales de los futuros usuarios. La prueba de este lamentable "método" rutinario la constituye la gran cantidad de diccionarios llamados de "traducción" que se parecen más bien a listados bilingües de términos "desnudos" por su falta de casi todos los datos necesarios para satisfacer las múltiples necesidades de sus usuarios.

El método más frecuente, "alternativo" al rutinario, es el uso de cuestionarios de diferente índole. Este método muestra el afán e interés del lexicógrafo en conocer las necesidades reales de sus usuarios potenciales, pero en la mayoría de los casos muestra también la falta de conocimiento de los muchos problemas y deficiencias, descritos tanto por lexicógrafos como por expertos de las ciencias sociales; cf. Hatherall (1984), Zikmund (1997), Hansen \& Andersen (2000), Welker (2006) y Tarp (2009).

Existen también otros métodos menos utilizados, como la observación directa o indirecta (p.ej. mediante log files) del proceso de traducción. Si la observación se combina con una posterior entrevista profunda, este método "objetivo" puede generar datos fiables y de gran relevancia sobre las necesidades lexicográficas que puedan producirse durante la traducción. Sin embargo, para tener un valor estadísticamente significativo requiere muchas observaciones.

A este respecto hay que distinguir entre el enfoque puramente comercial y el enfoque científico. Si el interés es solo reunir argumentos para una publicidad llamativa que permita la venta de gran cantidad de diccionarios (y la obtención de las correspondientes ganancias), una casa editorial puede conformarse con el conocimiento de las necesidades que tienen los usuarios en, 
digamos, el 90 o el 95 por ciento de las veces, o que ellos mismos creen que tienen. Por el contrario, si el interés es tener una idea real de todas las necesidades relevantes con el fin de concebir diccionarios que también ayuden a los usuarios a resolver sus problemas en uno de cada cien o de cada mil casos (ya que no tienen otro al que dirigirse), el lexicógrafo también debe aplicar métodos que le permitan descubrir estos casos.

Pongamos un ejemplo. La observación de traductores ejerciendo su profesión puede, sin duda ninguna, facilitar indicios importantes sobre las fases y subfases en las que pueden surgirles problemas. Sin embargo, para llegar a ser estadísticamente significativa, es decir, para producir un cuadro completo donde no se ignore ninguna subfase o tipo de problema que quizás no sea tan frecuente, aunque relevante para el trabajo lexicográfico, este método requiere un gran número de observaciones a diferentes traductores, por lo que es al mismo tiempo muy costoso y necesita mucho tiempo.

La teoría funcional recomienda un método más sencillo llamado de deducción, descrito detalladamente por Fuertes Olivera \& Tarp (2014). La deducción como método lexicográfico se basa en una estrecha colaboración entre un experto en lexicografía teórica y práctica y otro experto conocedor del proceso de traducción. Con este método, los dos trabajan juntos analizando todas las fases y subfases del proceso con el fin de determinar (deducir) dónde pueden surgir necesidades lexicográficamente relevantes y de qué tipos.

El experto podría, por ejemplo, ser un profesor universitario de traducción especializada con muchos años de experiencia. Si este profesor, a lo largo de su carrera, ha corregido miles de tareas de traducción y leído gran cantidad de protocolos en los que los estudiantes han explicado los pasos que han dado para buscar información que les permita traducir palabras, términos y expresiones específicos, y si este profesor a continuación ha discutido los respectivos protocolos con sus estudiantes, estará en una muy buena posición para determinar tanto las fases y subfases relevantes como los correspondientes tipos de necesidades.

De hecho, el material en que se basa para hacer este trabajo es, en la mayoría de los casos, mucho más amplio y representativo que el material conseguido con unos cuantos centenares de cuestionarios de dudosa calidad o unas decenas de observaciones y entrevistas. Además, el método descrito es mucho más fácil, barato y ahorra más tiempo que los otros métodos mencionados y produce, por regla general, datos no menos importantes y relevantes para el trabajo lexicográfico.

En el Centro de Lexicografía de la Universidad de Aarhus se han concebido decenas de proyectos lexicográficos aplicando este método y, generalmente, 
con muy buenos resultados. Son siempre proyectos que luego se han llevado a cabo -no para producir los patéticos "prototipos" que emplean grandes sumas de dinero sin llegar a terminarse- sino para producir y publicar diccionarios especializados reales.

\section{Fases y subfases de la traducción desde una perspectiva lexicográfica}

Es evidente que las fases y subfases del proceso de traducción pueden variar de traductor a traductor, de tarea a tarea. Pero también es evidente que un diccionario de traducción bien concebido debe cubrir todas las fases relevantes para el tipo -o los tipos- de usuarios previstos, y que el papel de la teoría es prestar ayuda y funcionar como guía en este sentido. Con tal fin, Tarp (2013) ha propuesto el siguiente esquema preliminar con tres fases fundamentales y una serie de subfases que abarcan todo el proceso de traducción en sentido amplio desde una perspectiva lexicográfica:

1. una fase de pretraducción en la que el traductor

a. prepara la traducción estudiando textos relevantes para obtener una idea general sobre la especialidad, la disciplina o el campo de conocimiento en cuestión,

b. lee todo el texto en la lengua de partida;

2. una fase de traducción (en el sentido estricto de la palabra) en la que el traductor

a. lee segmentos específicos del texto en la lengua de partida,

b. transfiere segmentos específicos del texto a la lengua de llegada,

c. reproduce segmentos específicos del texto en la lengua de llegada;

3. una fase de postraducción en la que el traductor (u otra persona)

a. revisa el texto traducido.

En todas estas fases y subfases, el traductor puede tener diversos tipos de necesidades cuya satisfacción requiere tanto tipos específicos de datos lexicográficos como tipos específicos de vías de acceso a estos datos. Antes de profundizar en esta discusión vamos a ofrecer una caracterización de las diferentes categorías de traductores para tener una idea más detallada de lo que puede necesitar cada una de ellas.

\section{Perfil y características del usuario}

Las necesidades relevantes para la lexicografía dependen, evidentemente, de las características del grupo usuario. Un tipo de usuario con características específicas no tiene necesariamente las mismas necesidades que otro tipo de 
usuario con otras características, incluso cuando están realizando el mismo tipo de tarea. Un usuario potencial de un producto lexicográfico tiene un sinfín de características de las cuales solo unas cuantas son lexicográficamente relevantes (por ejemplo, no es relevante para la lexicografía que una persona sea pelirroja, zurda, colérica, etc.) y de aquellas son aún menos relevantes en cada tipo de situación o actividad en que pueden producirse necesidades relevantes. En este sentido, para determinar las características lexicográficamente relevantes en lo que se refiere a la traducción especializada y trazar un perfil del grupo usuario previsto hay varios parámetros que deben tomarse en cuenta, de los cuales los más importantes son:

- conocimiento de la especialidad (que va a cubrir el diccionario)

- conocimiento comparativo de la especialidad (cuando hay diferencias culturales)

- destrezas y experiencia en la traducción

- destrezas lingüísticas generales en la lengua de partida

- destrezas lingüísticas generales en la lengua de llegada

- conocimiento de la terminología en la lengua de partida

- conocimiento de la terminología en la lengua de llegada

- conocimiento de las convenciones estilísticas en la lengua de partida

- conocimiento de las convenciones estilísticas en la lengua de llegada.

Aunque constituyen un continuo, las características basadas en estos parámetros pueden graduarse en baja, intermedia y alta (lego, semiexperto y experto) en función de la persona específica que realiza una traducción especializada. Desde el punto de vista de su formación y experiencia hay tres categorías fundamentales de traductores de textos especializados:

1. el traductor graduado en traducción especializada,

2. el estudiante de traducción especializada,

3. el experto de la especialidad que realiza traducciones.

Cada una de estas categorías de traductores tiene sus características específicas, determinando los tipos de necesidades que puede tener en las diversas fases y subfases del proceso de traducción y, por lo tanto, los tipos de datos y vías de acceso que permiten la satisfacción de estas necesidades.

Los traductores graduados pueden subdividirse en dos tipos: los que se han especializado dentro de un campo específico (por ejemplo, la traducción jurídica) y los que son traductores "multicampo", o sea, sin especialización dentro de un campo específico. Ambos tipos tienen, por definición, destrezas de traducción y lingüísticas generales en ambas lenguas, pero se diferencian 
considerablemente en lo que se refiere a su conocimiento de la especialidad, la terminología y las convenciones estilísticas correspondientes (cf. Nielsen 2010). Los primeros deben tener un conocimiento bastante bueno de la terminología y convenciones estilísticas, además de un conocimiento de la especialidad que les califica como semiexpertos. Por el contrario, los últimos son traductores legos con un conocimiento mínimo de la especialidad, la terminología y las convenciones estilísticas en ambas lenguas.

Los estudiantes de traducción especializada tendrán probablemente destrezas lingüísticas generales de niveles alto y medio en las lenguas en cuestión. Por otro lado, se caracterizarán por destrezas de traducción insuficientes (pero en crecimiento) combinadas con un bajo conocimiento de la especialidad, de su terminología y de sus convenciones estilísticas.

Los expertos de la especialidad que se dedican a hacer traducciones abarcan un amplio espectro de características. Todos ellos tendrán sin duda un alto grado de conocimiento de la terminología y de las convenciones estilísticas en cuestión, pero no necesariamente en ambas lenguas. Además, algunos de ellos tendrán destrezas de traducción avanzadas, en tanto que otros tendrán destrezas similares a las de los estudiantes ya mencionados. Como regla general, si un diccionario especializado está diseñado para ayudar tanto a traductores graduados como a estudiantes de traducción también cubrirá el amplio espectro de necesidades lexicográficas que pueden tener los expertos cuando realizan traducciones relacionadas con su especialidad. Lo único que los expertos no necesitan y que los distingue de las otras dos categorías de traductores es información básica sobre la especialidad.

\section{Posibles necesidades en las diversas fases}

Según la teoría funcional, una obra lexicográfica puede tener cuatro categorías fundamentales de funciones, o sea, 1) las comunicativas, donde se trata de ayudar a usuarios que encuentran problemas en diversas situaciones comunicativas (como la producción, recepción, traducción, revisión y corrección de textos); 2) las cognitivas, donde se trata de ayudar a usuarios que necesitan o desean aumentar su conocimiento de algo; 3 ) las operativas, donde se trata de ayudar a usuarios que necesitan consejos o instrucciones para realizar alguna acción física o mental no relacionada directamente con la comunicación; y 4) las interpretativas, donde se trata de ayudar a usuarios que necesitan interpretar algún signo, símbolo, etc. no lingüístico; cf. Tarp (2008) y Fuertes Olivera \& Tarp (2014). En su discusión de los manuales de instrucciones, Rodríguez Gallardo (2013) propone incluso una quinta categoría, las evaluativas, pero es todavía pronto para determinar si tiene relevancia para los diccionarios. 
Sin embargo, solo dos de las citadas categorías son relevantes en lo que se refiere a la traducción de textos especializados: las funciones cognitivas cuando se trata de ayudar al usuario que necesita información de fondo sobre la especialidad en cuestión, y las funciones comunicativas cuando se trata de ayudar al usuario en la lectura (recepción), transferencia, reproducción y revisión de textos. A este respecto cabe destacar que las necesidades cognitivas no solo pueden producirse en la fase de pretraducción, en la que el usuario puede necesitar información general sobre la especialidad, sino también en las fases de traducción y de postraducción, en las que la falta de conocimiento específico de la especialidad puede distorsionar y dificultar el proceso traductivo. A este respecto, Nielsen (2013) ha puesto de manifiesto que los traductores precisan de estos conocimientos cognitivos para poder traducir textos relacionados con la contabilidad. A continuación, discutiremos los diversos tipos de datos lexicográficos que pueden necesitar los traductores en las fases de pretraducción, traducción y postraducción, respectivamente.

\subsection{Fase de pretraducción}

En esta fase, los traductores pueden necesitar:

1. una introducción general y sistemática a toda o parte de la especialidad o disciplina de que trata el texto;

2. definiciones de términos específicos de la lengua de partida;

3. explicaciones de palabras y expresiones generales de la lengua de partida;

4. información de fondo sobre diversos fenómenos, procesos, cosas y aspectos relacionados con el texto.

Comentario: aquí cabe destacar que no hace falta una solución bilingüe para acceder a los datos lexicográficos correspondientes, que perfectamente pueden suministrarse en un diccionario monolingüe de la lengua de partida, solución que incluso es la mejor cuando la lengua de partida también es la lengua materna del traductor. Además, es importante notar que el punto 3, por regla general, solo es relevante si la lengua de partida no es la materna del traductor, y que en la mayoría de los casos no hace falta que se resuelva en un diccionario especializado si ya existen diccionarios no especializados que explican las palabras y expresiones generales. Finalmente, cabe mencionar que el punto 4 solo es relevante para los estudiantes y traductores graduados, pues se presupone que los expertos de la especialidad ya poseen los conocimientos necesarios en este sentido. 


\subsection{Fase de traducción}

En esta fase, los traductores pueden necesitar:

1. información de fondo específica;

2. definiciones de términos de la lengua de partida;

3. equivalentes de términos, colocaciones y expresiones fijas;

4. equivalentes de palabras y expresiones generales;

5. información sobre ortografía, género, flexión, propiedades sintácticas y convenciones estilísticas en la lengua de llegada.

Comentario: de todos los tipos de datos lexicográficos mencionados, solo el suministro de equivalentes ( 3 y 4) precisa de una solución bilingüe en tanto que esta solución es opcional para los otros tipos de datos (además, como en la fase anterior, los datos de tipo 4 no deben necesariamente tratarse en un diccionario especializado). Los datos de tipo 1 y 2 también pueden suministrarse en un diccionario monolingüe de la lengua de partida; los datos de tipo 5 pueden presentarse a) en un diccionario bilingüe de la lengua de partida a la de llegada, b) en un diccionario monolingüe de la lengua de llegada, o c) en un diccionario bilingüe de la lengua de llegada a otra lengua. A menudo, los traductores -y especialmente los que tienen experiencia, cf. sección 6- no tienen ningún problema en las subfases de lectura y transferencia del texto, sino solo cuando tienen que reproducirlo en la lengua de llegada (especialmente si no es su lengua materna). En casos como este, una solución bilingüe no sería capaz de ayudar a los traductores, a menos que reconstruyan todo el proceso traductivo desde la lectura del texto en la lengua de partida, una reconstrucción que obliga a utilizar mucho tiempo, por lo que no se puede esperar que los traductores la hagan.

\subsection{Fase de postraducción}

No deja de sorprender que esta fase en la que se realiza la revisión del texto traducido - por parte del propio traductor u otra persona ajena- sea una de las menos estudiadas en la literatura lexicográfica, especialmente si se tiene en cuenta que la revisión de textos es un proceso que se repite millones de veces cada día; cf. Tarp $(2004,2008)$. Un estudio detallado sobre las complejas necesidades lexicográficas que pueden tener las personas que realizan trabajos de revisión de textos traducidos muestra que hay seis subfases lexicográficamente relevantes, a saber: 1) recepción del texto original; 2) recepción del texto traducido; 3) evaluación de la transferencia; 4) corrección de la transferencia; 5) evaluación del texto traducido; y 6) corrección del texto traducido. A este respecto, Tarp (2007: 248) comenta: 
Sin profundizar en cada una de estas subfases que se combinan y repiten según los hábitos de trabajo de cada actor, cabe subrayar que gran parte de las necesidades relativas a estas subfases son las mismas como las que tienen los traductores en las diferentes fases del proceso de traducción, pero como el texto a corregir o revisar está escrito en la lengua de destino y también precisan de entender este texto, tienen relativamente más necesidades relacionadas con esta lengua, necesidades que por su naturaleza solo pueden cubrirse con un diccionario, monolingüe o bilingüe, que parte de la lengua de destino.

Por consiguiente, en esta fase compleja de revisión, los traductores o revisores del texto pueden necesitar:

1. información de fondo específica;

2. definiciones de términos de la lengua de partida;

3. explicaciones de palabras y expresiones generales de la lengua de partida;

4. equivalentes de términos, colocaciones y expresiones fijas;

5. definiciones de términos de la lengua de llegada;

6. información sobre ortografía, género, flexión, propiedades sintácticas y convenciones estilísticas en la lengua de llegada.

Comentario: la satisfacción de estas complejas necesidades requiere una combinación de soluciones lexicográficas. Los datos de tipo 1, 2 y 3 pueden suministrarse en una solución monolingüe de la lengua de partida (mejor solución si también es la lengua materna del traductor o revisor) o en una solución bilingüe de la lengua de partida a la de llegada (mejor solución si esta última es la lengua materna del traductor o revisor). Los datos de tipo 4 requieren necesariamente una solución bilingüe de la lengua de partida a la de llegada, en tanto que los de tipo 5 y 6 pueden suministrarse tanto en una solución monolingüe de la lengua de llegada como una bilingüe que parte de esta lengua.

\subsection{Consecuencias}

Las anteriores reflexiones tienen grandes consecuencias para la teoría y práctica de diccionarios de traducción. Sin embargo, antes de pasar a discutir estas consecuencias puede ser interesante analizar brevemente los resultados de varios estudios sobre traductores y su uso de diccionarios para ver si confirman o no estas reflexiones.

\section{Indicaciones generadas mediante otros métodos}

Gran parte de los estudios realizados sobre el comportamiento lexicográfico de los traductores adolece de varios tipos de problemas. Primero, en la 
mayoría de los casos se trata de estudios sobre las necesidades que tienen los traductores en relación con la traducción de textos no especializados. Segundo, las necesidades no han sido analizadas tal como se generan directamente en el proceso traductivo, sino solo como se reflejan indirectamente en consultas de diccionarios o bien como los mismos traductores las interpretan. Tercero, los estudios tienen generalmente muy pocos informantes, por lo que carecen de valor estadísticamente significativo, aunque ofrezcan "un exceso de porcentajes y decimales para documentar con qué frecuencia los informantes usan uno u otro diccionario" (Tarp 2009: 292).

Sin embargo, si lo que interesa no es tanto la cantidad de veces que los traductores buscan una u otra cosa, sino las necesidades que puedan tener por lo menos una o pocas veces, es decir, también en una de cada cien, de cada mil o de cada millón de consultas, entonces se puede abrir camino entre los muchos porcentajes y decimales para ver si en algunos de los estudios publicados hay indicios en tal sentido. A continuación, vamos a extraer información de cinco estudios dedicados al uso de diccionarios en relación con la traducción especializada; cf. Tomaszcyk (1989), Duvå et al. (1992), Mackintosh (1998), Varantola (1998) y Nord (2002). Todos estos estudios tienen desde un solo informante hasta unas pocas decenas, por lo que carecen de cualquier valor estadísticamente significativo. Sin embargo, esto no excluye que se saque información cualitati$v a$ de ellos para confirmar o no las reflexiones del apartado anterior.

En primer lugar, estos estudios comprueban que los traductores a menudo tienen problemas no solo relativos a los términos y expresiones especializados, sino también en lo que se refiere al vocabulario general.

En segundo lugar, los estudios también ponen en evidencia que los traductores con gran frecuencia consultan no solo diccionarios bilingües, sino también monolingües, tanto en la lengua de partida como la de llegada, lo que indica que no solo tienen problemas en la subfase de transferencia, sino también en las demás subfases descritas más arriba.

Especialmente interesante en este sentido es el estudio del polaco Jerzy Tomaszcyk (1989) quien, al hacer él mismo una traducción del polaco al inglés de un texto sobre el uso industrial de diamantes, escribió un protocolo en el que registró todas las consultas que hizo. De este protocolo se desprende que el también lexicógrafo polaco, con veinte años de experiencia como traductor, casi la mitad de las consultas no las hizo para buscar "algo nuevo" sino que las hizo para "confirmar mis propias predicciones", por lo que gran parte de las consultas se hicieron en diccionarios monolingües. Tomaszcyk (1989: 179) concluye diciendo que "como lo que uno busca en estos casos son datos en L2 (más bien que datos en L1 y sus equivalentes en L2), se desprende que, al hacer una traducción L1-L2, uno puede llegar muy lejos sin un diccionario L1-L2". 
En tercer lugar cabe destacar que todos estos estudios (con la posible excepción de Mackintosh (1998) que categoriza sus datos de otra forma) ponen de manifiesto que los traductores no solo tienen necesidades comunicativas relacionadas con el proceso traductivo sino también cognitivas, lo que se desprende de las muchas consultas en diccionarios categorizados como enciclopédicos. Por ejemplo, Duvå et al. (1992: 132) concluyen que sus informantes (todos ellos estudiantes de traducción) sufren de cuatro "incertidumbres", entre ellas la "incertidumbre sobre la materia" y el "lugar de las palabras en el universo". Las conclusiones que saca Krista Varantola de su estudio son también interesantes:

Very often in the case of translators the information needs are deeper, covering longer contexts and specialised "world knowledge" that does not belong in a dictionary: these complicated and interdependent needs tend to merge into each other, making it difficult if not impossible for a single reference work, however complex, to satisfy them. (Varantola 1998: 339)

La lexicógrafa finesa escribió este comentario en un momento en el que los diccionarios digitales todavía estaban en su primerísima fase. Con la experiencia acumulada desde entonces, hoy en día la situación es bien diferente. Más adelante veremos cómo no solo es posible sino también recomendable que una nueva generación de diccionarios de traducción en línea satisfaga el gran abanico de necesidades de información que puedan tener sus usuarios previstos. Pero antes vamos a sacar unas conclusiones de la discusión anterior para acercarnos aún más a un concepto más global de lo que puede y debe ser un diccionario de traducción en el siglo XXI.

\section{Esquema completo con las fases y subfases relevantes para la lexicografía}

En la sección 4 presentamos un esquema preliminar indicando las fases y subfases relevantes desde una perspectiva lexicográfica. Ahora, después de la discusión en las secciones 5 y 6 , podemos matizar aún más y presentar el siguiente esquema que muestra las actividades relacionadas con las tres fases fundamentales del proceso traductivo en las que los traductores pueden tener necesidades de tipo cognitivo y comunicativo:

1. La fase de pretraducción

a. estudio general sobre la materia o especialidad

b. recepción del texto en la lengua de partida

c. estudio específico de un tema relacionado con la materia.

2. La fase de traducción

a. recepción del texto en la lengua de partida 
b. transferencia del texto de la lengua de partida a la de llegada

c. reproducción del texto en la lengua de llegada

i. con problemas en las subfases anteriores

ii. sin problemas en las subfases anteriores

d. estudio específico de un tema relacionado con la materia.

3. La fase de postraducción

a. revisión del texto traducido

i. recepción del texto en la lengua de partida

ii. recepción del texto en la lengua de llegada

iii. evaluación de la transferencia

iv. corrección de la transferencia

v. evaluación del texto traducido

vi. corrección del texto traducido

b. estudio específico de un tema relacionado con la materia.

Un diccionario que está diseñado para ayudar a los traductores de textos especializados debe ser capaz de resolver todos los problemas y demás necesidades lexicográficamente relevantes que aquellos tengan al realizar las diversas actividades relacionadas con el proceso global de traducción. Como se desprende de la discusión anterior, no es cuestión solamente de incorporar los datos lexicográficos correspondientes, sino también de asegurar la vía de acceso más fácil a estos datos. Es una cuestión que tiene gran importancia para el concepto global de lo que debe ser un diccionario de traducción no solo de nombre sino también de hecho.

\section{Vías de acceso y concepto global de un diccionario especializado de traducción}

En los estudios sobre el uso de diccionarios discutidos en la sección 6, los informantes consultaron varios tipos de diccionarios monolingües y bilingües en ambas lenguas y hasta en ambas direcciones. Tomaszcyk (1998), por ejemplo, informa que consultó diccionarios monolingües de inglés y bilingües de polaco-inglés, inglés-polaco, ruso-inglés e inglés-ruso. Hasta cierto punto, algunas de estas consultas podrían deberse a la falta de un diccionario más conveniente, lo que debe ser el caso de los diccionarios ruso-inglés e inglés-ruso consultados. Para buscar una lógica mayor, a continuación vamos a discutir qué tipo de diccionario sería el más conveniente para resolver los diferentes tipos de necesidades que pueden producirse en las diversas fases y subfases del proceso de traducción cuando también se toma en cuenta la lengua materna del usuario. 


\subsection{Las mejores opciones}

En el subapartado 5.1 vimos cómo los traductores legos pueden necesitar una introducción general y sistemática a toda o a parte de la especialidad o disciplina cuando están en la fase de preparación de la traducción de un texto especializado. La mayoría de los diccionarios especializados llamados de traducción ignoran por completo esta importante necesidad de sus usuarios, mientras otros se conforman con una referencia a una o varias fuentes externas donde se puede obtener la información correspondiente. Tal solución no es mala, pero aún mejor sería la incorporación de una sección dedicada a este tema en el mismo diccionario, como han hecho Gubba $(1993,1995)$ en sus Juridisk ordbog dansk-tysk y Juridisk ordbog tysk-dansk, Kaufmann \& Bergenholtz (1998) en sus Diccionario enciclopédico de ingeniería genética español-inglés y Encyclopedic Dictionary of Gene Technology English-Spanish, y Fata (2005) en su Ungarisch-Deutsches, Deutsch-Ungarisches Fachwörterbuch zur Rentenversicherung. En este sentido, muchos traductores que no son expertos de la especialidad preferirían seguramente que la introducción estuviera escrita en su lengua materna, aunque también podría ofrecerse en ambas lenguas simultáneamente como han hecho Kaufmann \& Bergenholtz (1998). Esto es válido para la traducción tanto de la lengua materna como a la lengua materna. La correspondiente introducción sistemática podría suministrarse tanto en una solución monolingüe como en una solución bilingüe en una u otra dirección.

La definición de términos de la lengua de partida y la información de fondo sobre diversos fenómenos, procesos y cosas específicos que pueden necesitar los traductores en todas las fases debe ofrecerse también en su lengua materna. La mejor opción es suministrar los datos lexicográficos correspondientes en una solución monolingüe para la traducción desde la lengua materna (L1L2) y en una bilingüe para la traducción a la lengua materna (L2-L1). La segunda mejor opción sería una solución bilingüe en el primer caso y una monolingüe en el segundo, respectivamente.

Las explicaciones de palabras y expresiones generales de la lengua de partida que puede necesitar el traductor (o revisor) en las fases de pre- y postraducción son, por regla general, solamente relevantes para la traducción desde una lengua que no sea la materna. En la medida que el diccionario especializado se encargue de este problema, la mejor opción es suministrar estos datos en una solución bilingüe con equivalentes en la lengua materna, con lo que también se resuelve la necesidad, relativa a la subfase de transferencia, de ofrecer equivalentes de palabras y expresiones generales.

Los equivalentes de términos, colocaciones y expresiones fijas que se pueden necesitar en las fases de traducción y postraducción deben obligatoriamente 
colocarse en una solución bilingüe de la lengua de partida a la lengua de llegada para la traducción en ambas direcciones.

Como se argumentó más arriba, la necesidad de tener información sobre ortografía, género, flexión, propiedades sintácticas y convenciones estilísticas en la lengua de llegada puede producirse de dos formas diferentes en la subfase de reproducción de la fase central de traducción, o sea, cuando había y no había problemas en las subfases anteriores. En el primer caso, la mejor opción sería colocar los datos correspondientes en una solución bilingüe desde la lengua de partida a la de llegada, en tanto que la mejor opción en el segundo caso sería colocarlos en una solución monolingüe (o bilingüe) que parta de la lengua de llegada. Esta última solución también podría resolver el mismo tipo de necesidad cuando se produce en la fase de postraducción.

Finalmente, en el caso de la definición de términos de la lengua de llegada que el traductor o revisor puede necesitar en la fase de postraducción, la mejor opción es una solución monolingüe cuando la lengua de llegada también es su lengua materna, y una solución bilingüe desde la lengua de llegada a la lengua de partida (la materna).

\subsection{El mejor diseño global de un diccionario especializado de traducción}

Las reflexiones anteriores ponen en evidencia que un diccionario especializado de traducción debe ser algo más que un diccionario bilingüe si pretende responder a las muy variadas necesidades de sus usuarios en lo que se refiere tanto a los datos lexicográficos como a las vías de acceso a estos. De hecho, se desprende de lo anterior que el mejor diseño global de un diccionario concebido para ayudar a sus usuarios en la traducción de textos especializados de su lengua materna a una lengua extranjera debe tener tres componentes, a saber:

- un componente monolingüe de Ll

- un componente bilingüe de L1-L2

- un componente bilingüe de L2-L1.

De forma similar, el mejor diseño global de un diccionario concebido para ayudar a sus usuarios en la traducción de textos especializados de una lengua extranjera a su lengua materna debe tener dos componentes, a saber:

- un componente bilingüe de L2-L1

- un componente monolingüe de Ll.

En el universo impreso en el que hemos vivido hasta hace muy pocos años, la solución ideal sería publicar una serie de tres diccionarios para la traducción L1-L2 y otra serie de dos diccionarios para la traducción L2-L1. Sin embargo, 
como este tipo de diccionario especializado muy a menudo tiene un grupo de usuarios bastante reducido, tal solución no será atractiva para ninguna casa editorial a menos que el proyecto se apoye en fondos públicos. Por eso, una solución más pragmática -pero también basada en la teoría- sería optar por el segundo mejor diseño como también se discutió con anterioridad, o sea, una combinación de componentes L1-L2 y L2-L1 para la traducción L1-L2 y una combinación de componentes L2-L1 y L1 (o L1-L2) para la traducción L2-L1.

La experiencia con varios diccionarios combinados -por ejemplo, el ya mencionado Fachwörterbuch zur Rentenversicherung húngaro-alemán, alemán-húngaro, y el también mencionado Diccionario Enciclopédico de Ingeniería Genética español-inglés junto con su gemelo inglés-español, el Encyclopedic Dictionary of Gene Technology- muestra que este segundo mejor diseño, mediante unos pequeños ajustes y amplificaciones, podría materializarse en un diccionario combinado L1-L2/L2-L1. Estos tres diccionarios son ejemplos que ponen de manifiesto que tal solución no solo es viable, sino que también es de alta calidad (cf. Bergenholtz et al. 1994, Tarp 2005 y Fata 2009).

Sin embargo, a pesar de los méritos indiscutibles de estos y otros diccionarios similares, no es ningún secreto que adolecen de dos problemas sistémicos que no pueden resolverse de manera satisfactoria en el universo impreso. El primero de ellos son las búsquedas adicionales, que sus usuarios a menudo deben hacer en otras partes del diccionario para encontrar los datos requeridos. El segundo tiene que ver con la sobrecarga informativa, en el sentido de que el usuario en la mayoría de las consultas tropieza con cierta cantidad de datos superfluos e irrelevantes para el objetivo específico de la consulta. En ambos casos, estos problemas sistémicos pueden retrasar el proceso de consulta y hasta obstruir la recuperación de la información requerida en cada consulta específica. Estamos ante un problema inevitable en los diccionarios impresos -un problema, sin embargo, que fácilmente puede resolverse en el universo online-.

\section{Las nuevas posibilidades abiertas por los diccionarios en línea}

La paulatina transición al mundo digital, iniciada hace más o menos dos décadas, ha estado llena de promesas y desilusiones. Por un lado, la experiencia acumulada ya es más que suficiente para convencernos de que estamos ante una verdadera revolución no solo en lo que se refiere al nuevo medio de comunicación entre el lexicógrafo y su usuario, sino también, y aún más importante, a la promesa de alcanzar formas mucho más avanzadas de satisfacción individualizada de las necesidades de los usuarios. Por otro lado, no deja de sorprender el conservadurismo con que la mayoría de los lexicógrafos y casas 
editoriales se han lanzado al Nuevo Mundo Feliz, en referencia al famoso libro publicado por Aldous Huxley hace 80 años, cuando el mundo estaba ante otra gran revolución tecnológica.

Si uno estudia los diccionarios colocados en Internet durante los últimos años, se nota inmediatamente que los artículos que aparecen en la pantalla son copias casi al cien por cien de los artículos encontrados en los diccionarios impresos, bien porque son versiones digitales de diccionarios anteriormente publicados en papel, bien porque incluso los nuevos diccionarios digitales se conciben y producen sobre el molde de sus antepasados impresos. Poco se ha hecho para adaptarlos a las necesidades de los usuarios en cada tipo de situación o cada consulta específica (cf. Tarp 2011, 2012).

Evidentemente, la alternativa a esta lamentable situación no es dejarse arrastrar por las nuevas tecnologías y perder de vista a los usuarios y sus necesidades reales, sino aprovechar al máximo estas tecnologías bajo el control del lexicógrafo. En este sentido, Bothma (2011) ha mostrado cómo las tecnologías y técnicas digitales y de información desarrolladas hasta ahora ya permiten dar pasos importantes hacia diccionarios más adaptados a las necesidades específicas de sus usuarios previstos. Parece que lo que todavía impide a muchos lexicógrafos pasar plenamente al nuevo mundo feliz es la falta de una teoría avanzada de la lexicografía en la que puedan apoyarse para incorporar estas tecnologías y técnicas de la forma más adecuada.

Tarp (2011) y Fuertes Olivera \& Tarp (2014) indican dos tipos de diccionarios que pueden constituir el próximo y el futuro, respectivamente, a saber: el Modelo T Ford y el Rolls Royce, ambos apoyados en la teoría funcional de la lexicografía. La idea principal de estos dos modelos es evitar la sobrecarga informativa y acelerar el proceso de consulta ofreciéndoles a los usuarios acceso rápido a la mínima cantidad posible de datos lexicográficos, es decir, exactamente la cantidad (criterio cuantitativo) y tipos (criterio cualitativo) de datos necesarios para resolver sus necesidades específicas.

En el Modelo T Ford, los datos lexicográficos que aparecen en pantalla son únicamente los que están destinados a satisfacer las posibles necesidades (deducidas) en relación con cada función (determinada por actividad y tipo de usuario), en tanto que los Rolls Royces, aplicando técnicas más sofisticadas, ofrecen soluciones mucho más adaptadas a los usuarios individuales y sus necesidades específicas en cada consulta. Además, para asegurar la necesaria flexibilidad, ambos tipos de diccionarios también permiten que los usuarios "cambien de sillín" en medio del proceso de consulta si descubren que tienen necesidades adicionales (cf. Fuertes Olivera \& Tarp 2014). 
Los Modelos T Ford se basan en una serie de principios que pueden aplicarse de distintas maneras. Los Accounting Dictionaries que se componen de una serie de diccionarios monolingües y bilingües (danés, danés-inglés, inglés e inglés-danés) y los Diccionarios de Contabilidad (inglés-español, español y español-inglés) constituyen sendos ejemplos concretos de cómo estos principios pueden materializarse (cf. Fuertes Olivera \& Nielsen 2012 y Fuertes Olivera 2013). En los Diccionarios de Contabilidad (todavía en construcción en junio de 2013), se les ofrece inicialmente a los usuarios (que son principalmente hispanohablantes nativos) la opción de entrar en un componente español, español-inglés o inglés-español, donde pueden elegir entre los siguientes search methods (métodos de búsqueda) que les conducen casi instantáneamente a datos lexicográficos adaptados a su actividad específica:

Inglés-español

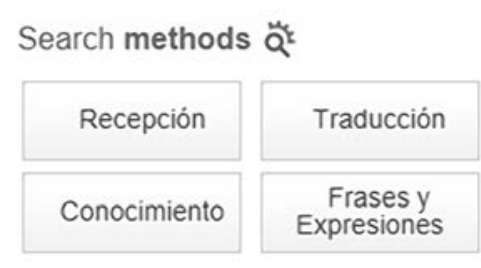

Español-inglés

Search methods ä

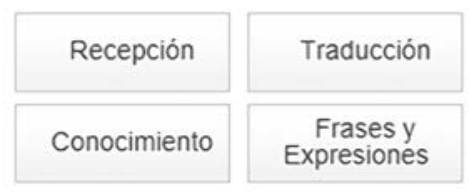

Español

Search methods ă:

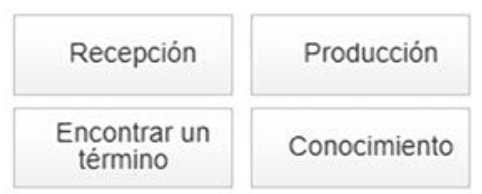

Figura 1: Métodos de búsqueda en los Diccionarios de Contabilidad 
Si el usuario entra en la parte español-inglés, teclea valor nominal en el campo de búsqueda y activa el botón Recepción aparecen los siguientes datos en la pantalla:

\author{
Recepción \\ valor nominal \\ Definition \\ El valor nominal o valor a la par es el valor con el que se emite un instrumento financiero, como un bono o una \\ acción, o una moneda. \\ face value
}

Figura 2: Datos generados por el botón "Recepción"

Si el usuario activa luego el botón Traducción aparecen otros datos relevantes:

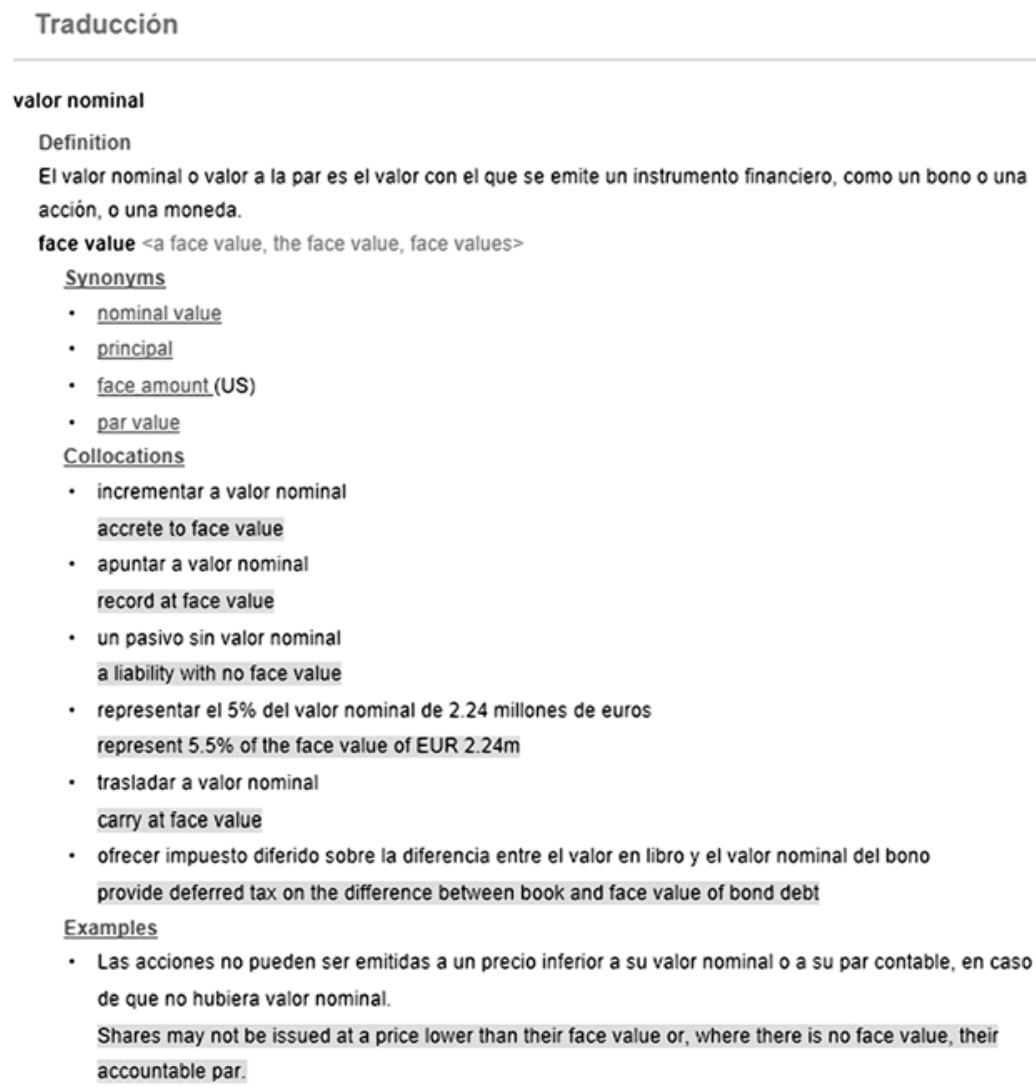

- ofrecer impuesto diferido sobre la diferencia entre el valor en libro y el valor nominal del bono provide deferred tax on the difference between book and face value of bond debt

Examples

- Las acciones no pueden ser emitidas a un precio inferior a su valor nominal o a su par contable, en caso de que no hubiera valor nominal.

Shares may not be issued at a price lower than their face value or, where there is no face value, their accountable par.

Figura 3: Datos generados por el botón "Traducción" 
Si el usuario activa el botón Frases y Expresiones aparecen los siguientes datos, muy requeridos por traductores de textos de contabilidad:

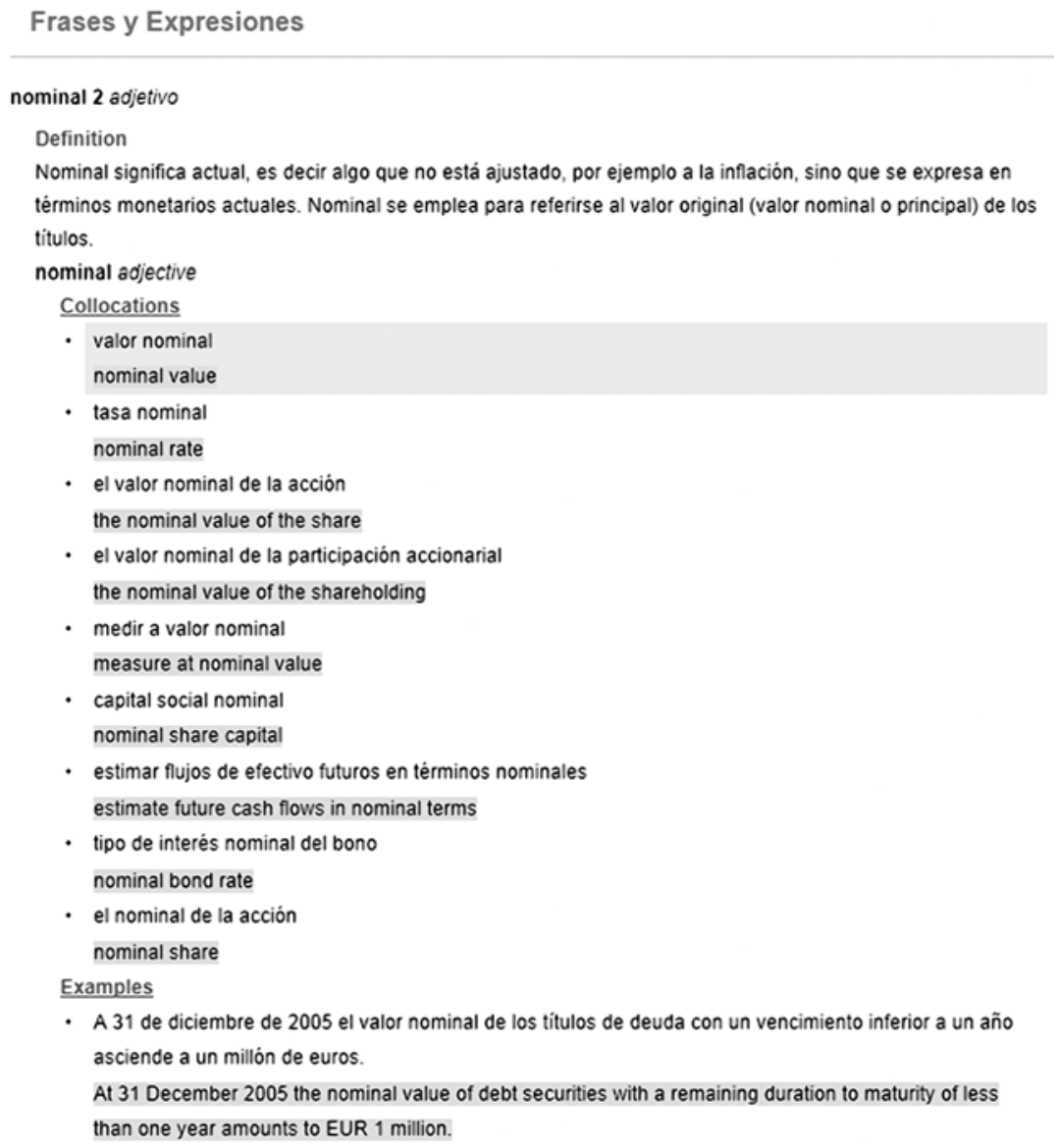

Figura 4: Datos generados por el botón "Frases y Expresiones"

Si el usuario finalmente activa el botón Conocimiento aparecen todos los datos anteriores juntos. Como ya se ha mencionado, los Diccionarios de Contabilidad todavía están en construcción (junio de 2013) y en un futuro próximo se añadirán otros componentes y detalles.

En otros Modelos T Ford sería beneficioso que los usuarios pudieran indicar al principio su lengua materna y actividad junto con otras características relevantes mediante distintas técnicas interactivas. Tal información podría 
entregarse o bien una vez para siempre cuando se usa el mismo diccionario, que la almacena para aprovecharla en futuras consultas (solución por defecto), o bien cada vez que se empieza una nueva tarea, por ejemplo la traducción de un texto especializado. Basándose en tal información, el sistema automáticamente calcula qué tipos de datos ha de suministrar al usuario en cada caso.

Si cognición representa la situación en la que el traductor precisa de información especializada sobre la materia o especialidad, las reflexiones de las secciones anteriores indican que el diccionario debe, por lo menos, ofrecerle las siguientes opciones de búsqueda según la actividad cuando empieza una consulta basada en L1 o L2 en relación con una traducción L1-L2 o L2-L1, respectivamente:

\begin{tabular}{|c|c|c|c|}
\hline Búsqueda & Traducción L1-L2 & Traducción L2-L1 & Combinación \\
\hline En Ll & $\begin{array}{c}\text { Cognición (L1) } \\
\text { Recepción (L1) } \\
- \\
\text { Transferencia (L1-L2) }\end{array}$ & $\begin{array}{c}\text { Cognición (L1) } \\
\text { Recepción (L1) } \\
\text { Reproducción (L1) } \\
-\end{array}$ & $\begin{array}{c}\text { Cognición (L1) } \\
\text { Recepción (L1) } \\
\text { Reproducción (L1) } \\
\text { Transferencia (L1-L2) }\end{array}$ \\
\hline En L2 & $\begin{array}{c}\text { Reproducción (L2-L1) } \\
\text { Cognición (L2-L1) } \\
\text { Recepción (L2-L1) } \\
-\end{array}$ & $\begin{array}{c}- \\
\text { Cognición (L2-L1) } \\
\text { Recepción (L2-L1) } \\
\text { Transferencia (L2-L1) }\end{array}$ & $\begin{array}{l}\text { Reproducción (L2-L1) } \\
\text { Cognición (L2-L1) } \\
\text { Recepción (L2-L1) } \\
\text { Transferencia (L2-L1) }\end{array}$ \\
\hline
\end{tabular}

Esquema 1: Posibles opciones de búsqueda en Modelos T Ford lexicográficos

El esquema 1 muestra que los diccionarios de traducción L1-L2 y los de traducción L2-L1 comparten la mayoría de las opciones de búsqueda que permiten el acceso a tipos de datos relacionados con la actividad del usuario. Por eso, resulta relativamente fácil combinar los dos tipos de diccionarios en un mismo proyecto. Aunque la gran mayoría de diccionarios en línea todavía no se califican como Modelos T Ford, el desafío hoy es dejar estos modelos atrás y dar pasos para una satisfacción más personalizada de las necesidades de los usuarios (Rolls Royces).

Para tal fin ya están disponibles varias técnicas, por ejemplo, áreas sensibles al ratón, ventanas pop-up, hipertextos y enlaces que permiten el acceso a datos suplementarios y adicionales. Otra posibilidad es el diseño del modelo de artículo por el propio usuario mediante técnicas interactivas para tener una presentación individualizada en la pantalla de los tipos de datos requeridos. Bothma (2011), Heid et al. (2012), Prinsloo et al. (2012) y Fuertes Olivera \& Tarp (2014) discuten varias de estas y otras técnicas que abren nuevos y grandes horizontes para la lexicografía online en general y la lexicografía de la traducción especializada en particular. 


\section{Conclusión}

Uno de los autores del Nordisk leksikografisk ordbok (Diccionario Nórdico de Lexicografía), que es el resultado de una cooperación entre lexicógrafos de varios países nórdicos, tuvo aparentemente un día infeliz cuando definió diccionario de traducción como "un diccionario concebido para la transferencia de un texto de una o varias lenguas a una o varias otras lenguas" (cf. Bergenholtz et al. 1997). Lamentablemente, ni el mejor diccionario de traducción puede transferir un texto de una lengua a otra; solo puede ayudar al traductor cuando este tiene ciertos tipos de problemas al realizar tal transferencia. No puede sustituir la experiencia y las destrezas de traducción desarrolladas durante muchos años de estudios y práctica. Si se entiende así, un diccionario puede llegar a ser una herramienta relevante e indispensable para el traductor. Jamás debe convertirse en un artefacto que le robe el tiempo creándole nuevas dudas, problemas y obstáculos.

En esta contribución hemos discutido algunos de los problemas relacionados con este tipo de herramienta y hemos indicado algunos caminos para ir superando los problemas y llegar a productos cada vez más útiles para los traductores. La idea de fondo de toda esta discusión ha sido la necesidad de desarrollar una teoría avanzada de los diccionarios especializados de traducción, pues sin tal guía se demorará aún más la producción de los diccionarios que necesitan y merecen los profesionales de la traducción por la noble faena a la que se dedican.

\section{Bibliografía}

BassnetT, Susan. (2001) Translation Studies. Third edition. London \& New York: Routledge.

BergennolTZ, Henning; Ilse Cantell; Ruth Vatvedt Fjeld; Dag Gundersen; Jón Hilmar Jónsson \& Bo Svensén. (1997) Nordisk leksikografisk ordbok. Oslo: Universitetsforlaget.

BERGENHOLTZ, Henning; Sven Tarp \& Uwe Kaufmann. (1994) "Vore mænd i Havanna: Udarbejdelse af konception til en spansk-engelsk genteknologisk ordbog." Hermes, Journal of Linguistics 13, pp. 291-304.

Bothma, Theo J. D. (2011) "Filtering and Adapting Data and Information in an Online Environment in Response to User Needs." En: Fuertes Olivera, Pedro A. \& Henning Bergenholtz (eds.) 2011. e-Lexicography: The Internet, Digital Initiatives and Lexicography. London \& New York: Continuum, pp. 71-102.

BurkHANOv, Igor. (1998) Lexicography. A Dictionary of Basic Terminology. Rzeszów: Wydawnictwo Wyzszejszkol y Pedagogicznej. 
DUVÅ, Grete; Anna-Lise Laursen \& Lisbet Maidahl. (1992) "Brugerundersøgelse vedrørende oversættelse af fagtekst.” En: Vatvedt Fjeld, Ruth (ed.) 1992. Nordiske studier i leksikografi. Rapport fra Konferanse om leksikografi i Norden 28.-31. mai 1991. Oslo: Nordisk forening for leksikografi, pp. 105-133.

FATA, Idilkó. (2005) Ungarisch-Deutsches, Deutsch-Ungarisches Fachwörterbuch zur Rentenversicherung. Szeged: Grimm Kiadó.

FATA, Idilkó. (2009) Das zweisprachige Translationswörterbuch für Fachsprachen in der wissenschaftlichen Theorie und Praxis. Budapest: Tinta Könyvkiadó.

Fuertes Olivera, Pedro A. (2013) "The theory and practice of specialised online dictionaries for translation." Lexicographica 29, pp. 69-91.

Fuertes Olivera, Pedro A; Henning Bergenholtz; Sandro Nielsen; Pablo Gordo Gómez; Lise Mourier; Marta Niño Amo; Ángel de los Ríos Rodicio; Ángeles Sastre Ruano; Sven Tarp \& Marisol Velasco Sacristán. (2013) Diccionarios de contabilidad. Hamburgo: Lemma.com.

Fuertes Olivera, Pedro A. \& Sandro Nielsen (2012) "Online dictionaries for assisting translators of LSP texts: the Accounting Dictionaries." International Journal of Lexicography 25:2, pp. 191-215.

Fuertes Olivera, Pedro A. \& Sven Tarp. (2014) Theory and practice of specialised online dictionaries: Lexicography versus terminography. Berlin \& New York: De Gruyter (en preparación).

GERZYMISCH-ARBOGAST, Heidrun; Gerhard Budin \& Gertrud Hofer (eds.) (2008) LSP Translation Scenarios. Selected Contributions to the EU Marie Curie Conference Vienna 2007. MuTra Journal. Thematic Volumes on Multidimensional Translation. Volume 2.

GuBBA, Wilhelm. (1993) Juridisk ordbog dansk-tysk. Copenhague: Gads Forlag. GUBBA, Wilhelm. (1995) Juridisk ordbog tysk-dansk. Copenhagen: Gads Forlag. HANSEN, Erik Jørgen \& Bjarne Hjort Andersen. (2000) Et sociologisk varktøj. Introduktion til den kvantitative metode. Copenhagen: Hans Reitzels Forlag.

HARTMAnN, Reinhard \& Gregory James. (2001) Dictionary of Lexicography. London \& New York: Routledge.

Hatherall, Glyn. (1984) "Studying dictionary use: Some findings and proposals.” En: Hartmann, R. R. K. (ed.) 1984. LEXeter '83 Proceedings. Papers from the International Conference on Lexicography at Exeter, 9-12 September 1983. Tübingen: Max Niemeyer, pp. 183-189.

HeID, Ulrich; D. J. Prinsloo \& Theo J. D. Bothma. (2012) "Dictionary and corpus data in a common portal: state of the art and requirements for the future." Lexicographica 28 , pp. 269-291.

KaUfmann, Uwe \& Henning Bergenholtz (eds.) (1998) Diccionario enciclopédico de ingeniería genética español-inglés. Toronto: Lugus.

KAUFMANN, Uwe \& Henning Bergenholtz (eds.) (1998) Encyclopedic Dictionary of Gene Technology. English-Spanish. Toronto: Lugus. 
MACKINTOSH, Kristen. (1998) "An Empirical Study of Dictionary Use in L2-L1 Translation.” En: Atkins, B. T. Sue (ed.) 1998. Using Dictionaries: Studies of Dictionary Use by Language Learners and Translators. Tübingen: Niemeyer, pp. 121-149.

MARello, Carla. (2003) “The Bilingual Dictionary.” En: Hartmann, R. R. K. (ed.) 2003. Lexicography. Critical Concepts II. London \& New York: Routledge, pp. 325-342.

NiELSEN, Sandro. (2010) "Specialized Translation Dictionaries for Learners." En: Fuertes Olivera, Pedro A. (ed.) 2010. Specialized Dictionaries for Learners. Berlin \& New York: De Gruyter, pp. 69-82.

NielSEN, Sandro. (2013) "Domain-specific knowledge in lexicography: how it helps lexicographers and users of accounting dictionaries intended for communicative usage situations." Hermes, Journal of Language and Communication in Business 50, pp. 51-60.

NiElSEn, Sandro; Lise Mourier \& Henning Bergenholtz. (2013) Accounting dictionaries. Odense: Ordbogen.com.

NORD, Britta. (2002) Hilfsmittel beim Übersetzen. Eine empirische Studie zum Rechercheverhalten professioneller Übersetzer. Frankfurt am Main: Peter Lang.

PiOTROWsKI, Tadeusz. (1994) Problems in bilingual lexicography. Wroclaw: Wydawnictwo Uniwersytetu Wroclawskiego.

PRINSLOO, D. J.; Ulrich Heid; Theo J. D. Bothma \& Gertrud Faaß. (2012) "Devices for Information Presentation in Electronic Dictionaries." Lexikos 22, pp. 290-320.

RODRíGUez GALLARDO, Ángel. (2013) "The function theory and its application on manuals of economics." Hermes, Journal of Language and Communication in Business 50, pp. 83-95.

SNell-HoRnBy, Mary; Franz Pöchhacker \& Klaus Kaindl (eds.) (1992) Translation studies: an interdiscipline. Amsterdam \& Philadelphia: John Benjamins.

TARP, Sven. (2004) "Korrektur og retning som leksikografiske funktioner." Hermes, Journal of Linguistics 33, pp. 117-147.

TARP, Sven. (2005) "The pedagogical dimension of the well-conceived specialised dictionary." Ibérica 10, pp. 7-21.

TARP, Sven. (2007) "¿Qué requisitos debe cumplir un diccionario de traducción del siglo XXI?" En: Fuertes Olivera, Pedro A. (ed.) 2007. Problemas lingüísticos en la traducción especializada. Valladolid: Universidad de Valladolid, pp. 227-256.

TARP, Sven. (2008) Lexicography in the borderland between knowledge and non-knowledge. Tübingen: Niemeyer.

TARP, Sven. (2009) "Reflections on lexicographic user research." Lexikos 19, pp. 275-296. 
TARP, Sven. (2011) "Lexicographical and Other e-Tools for Consultation Purposes: Towards the Individualization of Needs Satisfaction." En: Fuertes Olivera, Pedro A. \& Henning Bergenholtz (eds.) 2011. e-Lexicography: The Internet, Digital Initiatives and Lexicography. London \& New York: Continuum, pp. 54-70.

TARP, Sven. (2012) "Online dictionaries: today and tomorrow." Lexicographica 28, pp. 253-267.

TARP, Sven. (2013) "What should we demand from an online dictionary for specialized translation?" Lexicographica 29, pp. 146-164.

TOMASZCZYK, Jerzy. (1989) "L1-L2 Technical Translation and Dictionaries." En: Pöhl, Esther \& Mary Snell-Hornby (eds.) 1989. Translation and Lexicography. Papers Read at the Euralex Colloquium Held at Innsbruck, 2-5 July 1987. Amsterdam: John Benjamins, pp. 177-186.

VARANTOlA, Krista. (1998) "Translators and their Use of Dictionaries." En: Atkins, B. T. Sue (ed.) 1998. Using Dictionaries: Studies of Dictionary Use by Language Learners and Translators. Tübingen: Niemeyer, pp. 179-192.

Welker, Herbert Andreas. (2006) O uso de dicionários: Panorama geral das pesquisas empíricas. Brasilia: Thesaurus.

ZIkMUND, William G. (1997) Business Research Methods. Fort Worth: The Dryden Press / London: Harcourt Brace College Publishers.

\section{NOTA BIOGRÁFICA / BIONOTE}

El doctor Sven Tarp es traductor jurado y catedrático de español en la Escuela de Ciencias Empresariales y Sociales de la Universidad de Aarhus (Dinamarca), donde trabaja actualmente como Director de su Centro de Lexicografía. Es también catedrático extraordinario del Departamento de Afrikaans y Flamenco de la Universidad de Stellenbosch (Sudáfrica), miembro extraordinario del Centro Internacional de Lexicografía de la Universidad de Valladolid (España), y visitante distinguido de la Ciudad de Santiago de Cuba. Con un un doctorado ordinario en lexicografía especializada (1993) y un doctorado extraordinario por méritos investigadores sobresalientes en lexicografía para el aprendizaje (2006), es uno de los fundadores de la Teoría Funcional de la Lexicografía y ha publicado gran número de artículos en revistas y monografías. Es autor de Lexicography in the Borderland Between Knowledge and Nonknowledge (2008), coautor del Manual de Lexicografía Especializada: Teoría y Práctica (2014), editor de Fagleksikografi som speciale (1998), y coeditor del Manual of Specialised Lexicography (1995), Lexicography in the 21st Century (2009) y Lexicography at a Crossroads: Dictionaries and Encyclopedias today, 
Lexicographical Tools tomorrow (2009). Además, es editor o coautor de unos veinte diccionarios especializados.

Dr. Sven Tarp is a Sworn Translator and Professor of Spanish at the School of Business and Social Sciences at the University of Aarhus (Denmark), where he is currently the Head of its Centre for Lexicography. He is also Extraordinary Professor at the Department of Afrikaans and Dutch at the University of Stellenbosch (South Africa), Extraordinary Board Member of the International Centre for Lexicography at the University of Valladolid (Spain), and Distinguished Visitor of the City of Santiago de Cuba (Cuba). With a Ph.D. in specialised lexicography (1993) and a Doctor's Degree in learners' lexicography (2006), he is one of the founders of the Function Theory of Lexicography and has published a big number of contributions in journals and well-known series. He is the author of Lexicography in the Borderland Between Knowledge and Non-knowledge (2008), co-author of Theory and Practice of Specialised Online Dictionaries: Lexicography versus Terminography (2014), editor of Fagleksikografi som speciale (1998), and co-editor of Manual of Specialised Lexicography (1995), Lexicography in the 21st Century (2009), and Lexicography at a Crossroads: Dictionaries and Encyclopedias today, Lexicographical Tools tomorrow (2009). In addition, he is the editor or co-author of several specialised dictionaries. 